\title{
On the Impact of Salinity Barrier Layer on the Pacific Ocean Mean State and ENSO
}

\author{
Christophe Maes ${ }^{1}$ and Sophie Belamari ${ }^{2}$ \\ ${ }^{1}$ Laboratoire d'Etudes en Géophysique et Océanographie Spatiales, \\ Institut de Recherche pour le Développement (IRD), New Caledonia \\ ${ }^{2}$ Météo-France, Centre National de Recherches Météorologiques, Toulouse, France
}

\begin{abstract}
Observational studies of the western Pacific Ocean have suggested since the mid-1980s that the barrier layer resulting from the salinity stratification within the mixed layer could influence significantly the ocean-atmosphere interactions. Numerical experiments based on a CGCM are designed and analyzed in such a goal. The formation of the barrier layer is primarily identified as resulting from a tilting/shearing mechanism in which horizontal and vertical gradients of salinity, as well as the dynamical response of the ocean to westerly winds, are tightly coupled. When the contribution of salinity to the computation of the horizontal pressure gradient force in the ocean model is cancelled within the equatorial warm pool, both the mean climatology and the low frequency variability are affected as the result of a complete annihilation of the barrier layer. The decreased sensitivity of the coupling between the SST, winds and atmospheric deep convection is likely due to the deepening of the ocean mixed layer that cools the SST and weakens the amplitude of its variability. These local changes within the western Pacific warm pool also induce a basin scale response that weakens the amplitude of ENSO variability. These results suggest that the formation of the barrier layer is a key element of the whole Pacific ocean-atmosphere coupled system.
\end{abstract}

\section{Introduction}

Despite considerable progress in the representation of the tropical mean state and climate variability as simulated by coupled ocean-atmosphere general circulation models, CGCMs, (Delecluse et al. 1998), Collins et al. (2010) recently concluded that it is not yet possible to say whether the El Niño/Southern Oscillation (ENSO) activity will be enhanced or damped within the context of global warming. Accurate predictions of tropical and global climate variability, including ENSO, depend consequently on current model performance, and in particular, on the ability of CGCMs to understand and reduce their systematic errors in the time mean state.

In the Pacific Ocean, a stringent test for CGCMs is the representation of the mean zonal gradient in sea surface temperature (SST) at the equator. The simulated equatorial cold tongue generally tends to be too strong, too narrow, and to extend too far west. In consequence, warm waters of the so-called warm pool in the western equatorial Pacific region are misrepresented in most of CGCMs. Moreover, Maes et al. $(2002,2005)$ showed that such a region is crucial for the response simulated by their coupled model during the onset and heat buildup phases before the development of different ENSO events. They suggested in particular that the salinity stratification should be considered with care in climate modeling and forecasts, and that the feedbacks at work should require a fully coupled response of the ocean-atmosphere system.

In studying the thermodynamics of the ocean mixed layer in the western equatorial Pacific, Lukas and Lindstrom (1991) first noticed that isothermal layers were deeper than the mixed layer

Corresponding author: Christophe Maes, Laboratoire d'Etudes en Géophysique et Océanographie Spatiales, Institut de Recherche pour le Développement (IRD), BP A5 Noumea, 98848, New Caledonia. E-mail: christophe. maes@ird.fr. (C2011, the Meteorological Society of Japan. depth (MLD) itself. The MLD was determined using a density criterion instead of a criterion based solely on the temperature profile. They attributed this discrepancy to salinity stratification and suggested that entrainment cooling could not be an important component in the western Pacific warm pool heat budget. By way of calling attention to this mechanism, the distance between the bottom of the mixed layer and the top of the thermocline was named the barrier layer thickness (BLT). By analyzing ocean in situ profiles, Ando and McPhaden (1997) and, more recently, Bosc et al. (2009) both concluded that, for mixed-layer temperatures in the $25-30^{\circ} \mathrm{C}$ range, the presence of a salinity barrier layer significantly affects heat entrainment into the mixed layer, thereby impacting the SST and hence, the ENSO variability.

In this study, we intend to document specifically the impact of the barrier layer on the mean state and ENSO as simulated by a CGCM of the Pacific Ocean. This study aims to complete the previous results discussed by Maes et al. $(2002,2005)$. In exploring the role of the salinity barrier layer, the strategy involves two main steps. The first consists in diagnosing its pertinence in the dynamics of the warm pool and ultimately in ENSO as simulated by the coupled GCM. Second, the coupled model is modified in order to cancel out the formation of the BLT. Section 2 briefly describes the coupled model whereas Section 3 presents the main results. Section 4 concludes with a discussion.

\section{Model and experimental design}

The ocean-atmosphere coupled model considered in this study is the same one as used by Belamari et al. (2003) and by Maes et al. (2002, 2005). Briefly, the model consists of a global atmospheric GCM coupled to a tropical Pacific Ocean GCM. The horizontal resolutions of both models are relatively coarse, $3.75^{\circ}$ corresponding to a T31 triangular truncation for the atmosphere and $0.5^{\circ}$ in latitude by $1^{\circ}$ in longitude within the equatorial band for the oceanic model. The coupled domain extends from $30^{\circ} \mathrm{N}$ to $30^{\circ} \mathrm{S}$ but it is important to keep in mind that the model is completely free of damping from $15^{\circ} \mathrm{N}$ to $15^{\circ} \mathrm{S}$. More important, no flux correction is applied to the daily exchanges of SST, solar and net heat fluxes, surface wind stress and freshwater budget.

The control experiment starts in January with initial conditions provided by a previous forced experiment; the model is then integrated over a 70 -year period. The first 8 years are considered as a spin-up period and are discarded in the following analyses. The design of the perturbed experiment is based on the idea of removing the processes implied in the formation of the salinity barrier layer within the warm pool region. More details are given in the following section. The perturbed experiment starts in January of year- 9 of the control experiment and it is integrated independently over a 32-year period. During the first two years, the perturbed experiment exhibits a strong shift in the mean state that reaches another stable equilibrium hereafter. These first two years are thus discarded and the following comparisons focusing on the mean state and ENSO variability are based on a 30-year long period for each experiment.

\section{Results}

Before analyzing the response of the coupled model to the modifications applied to the model, it is important to determine the 
physical processes that are involved in the BL formation. Lukas and Lindstrom (1991) proposed the hypothesis that the barrier layer may result from the subduction of saltier mixed-layer waters originating from the central Pacific below the fresh mixed layer of the warm pool. In studying migrations of the warm pool, Picaut et al. (1996) and Maes et al. (2004) showed that the eastern edge of the warm pool is rather characterized by a zonal convergence of water masses and by a salinity front: When the warm pool moves eastward over the central Pacific under the influence of a vertically sheared eastward jet, the salinity front is tilted over the vertical resulting in the formation of a barrier layer with fresh waters from the west overlaying saltier water from the east. Roemmich et al. (1994) referred to this mechanism as a tilting/shearing mechanism, and proposed that the zonal salinity gradient may be as important as the wind stress in driving the surface jet. Cronin and McPhaden (2002) illustrated how a thick and long-lived barrier layer would result from such oceanic response to westerly wind burst.

To examine further the relationships between the barrier layer and various oceanic conditions in the control experiment, lagged correlations are calculated along the vertical on a 5-day average basis, for longitudes ranging from $160^{\circ} \mathrm{E}$ up to $180^{\circ}$, that corresponds roughly to the warm pool. Local correlations at $165^{\circ} \mathrm{E}$ of the BLT with the zonal current anomalies on the one hand, and with the anomalies of the salinity contribution to the pressure gradient on the other hand are shown in Fig. 1 (results are similar for other longitudes). The BLT exhibits strong positive (negative) correlations with the zonal current anomalies at the surface (subsurface) whereas no significant correlation is found with the meridional current (not shown). At the surface, eastward current anomalies lead the BLT by 10 to 15 days, indicating that the occurrence of an eastward surface jet induces the formation of a barrier layer in the following 10-day period (tilting and advection mechanisms). Consistently, anomalies of the salinity contribution to the zonal pressure gradient lead the BL formation by a few days. Similar lagged correlations between the zonal wind stress and the oceanic conditions (not shown) confirm that these lags are consistent with the time necessary for a westerly wind burst to drive the local ocean surface response, to induce a positive zonal pressure gradient, and lastly, to fully develop a sheared eastward jet. As demonstrated by Maes et al. (2002, 2005), such dynamics is crucial for the onset and buildup periods simulated by the present coupled model. All the aforementioned points result on longer periods in the formation of a barrier layer due to both zonal advection and tilting/shearing mechanism following the same processes as described by Roemmich et al. (1994). Conversely, the correlation between BLT and subsurface zonal current is negative and maximal near $140 \mathrm{~m}$ at the zero lag. This only indicates that the BL formation is in phase with the equatorial undercurrent deceleration associated with the zonal pressure gradient positive anomaly.
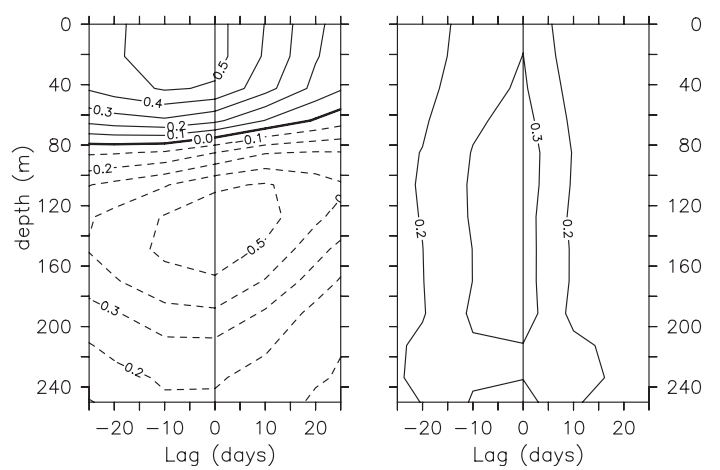

Fig. 1. Lagged correlation coefficient between the BLT and anomalies of (left) zonal current and (right) contribution of the salinity field to the zonal pressure gradient force as a function of depth at $0^{\circ} \mathrm{E}-165^{\circ} \mathrm{E}$. A positive lag implies that BLT variability occurs prior to the field anomaly. Correlations are computed over a 70 -yr period of time and values larger than $+/-0.3$ are significant at the $95 \%$ confidence interval.
Based on such analyses, the perturbed experiment is then obtained by removing the salinity contribution only into the computation of the pressure horizontal gradient within the equatorial warm pool. Specifically, within the $4^{\circ} \mathrm{N}-4^{\circ} \mathrm{S}$ band and for grid points where SST is warmer than $28^{\circ} \mathrm{C}$, the salinity field is changed to a constant value of 35 in the computation of the horizontal pressure gradient field instead of using the salinity vertical profile. This artifice only affects the determination of the pressure gradient whereas the salinity field remains free to evolve. Note that the horizontal pressure gradient is minimally modified by this procedure, as the main contribution due to temperature remains unchanged.

The design of the perturbed experiment is successful in the sense that the BLT disappeared within a period of less than one year and it did not recover thereafter. Changes in the time mean state of the perturbed experiment are evident at the basin scale, both in the atmosphere and in the ocean. These changes are documented first as they are important components that explain the low frequency variability of the perturbed experiment. Figure 2 shows the 30-yr mean sea surface salinity (SSS), SST and the MLD fields for the control and perturbed experiments. In the perturbed experiment, the SSS is nearly constant along the equatorial band, and the salinity front associated with the fresh warm pool almost disappears (Fig. 2a). Outside of the equatorial band, the general structure of main features remains basically unchanged and the small differences could be likely explained by the atmospheric response. The lack of horizontal gradient in surface salinity also extends down into the upper layers of the ocean. It means that, after the spinup period when the model climatology had changed, the conditions that would have enabled the recovery of the barrier layer are no longer possible in the perturbed experiment.

Similarly to the previous experiments (Maes et al. 2002, 2005), the removal of the salinity stratification within the warm pool results in both local and basin-scale responses in the oceanatmosphere system. Figure $2 \mathrm{~b}$ shows that the SSTs over the warm pool decrease by about $0.5^{\circ} \mathrm{C}$ and become slightly cooler than $28^{\circ} \mathrm{C}$. Even if this change is modest, it is sufficient to induce the displacement of the atmospheric main convective activity toward the far western Pacific Ocean as inferred from the planetary-scale divergent circulation (Fig. 3). In the control experiment, the main center of convective activity is localized near the equator around $140^{\circ} \mathrm{E}$ while shifted to the vicinity of the Philippines archipelago in the perturbed experiment. The amplitude of the divergent circulation has also decreased by $40 \%$ approximately. These changes in the convective activity explain the decrease in the freshening of the waters throughout the equatorial region in the perturbed experiment when compared to the control experiment. Such reduction in the ascending and descending branches of the Pacific Walker circulation induces weaker equatorial trade winds, leading to warmer waters in the eastern Pacific $\left(+1.5^{\circ} \mathrm{C}\right.$ near $\left.100^{\circ} \mathrm{W}\right)$ associated with a decrease in the basin-scale SST zonal gradient (Fig. 2b).

Such changes in the surface fields also reflect modifications that occur simultaneously in the upper ocean layers with a significant deepening of the mixed layer (Fig. 2c). The most drastic changes are seen in the western Pacific where the depth of the MLD has roughly doubled. This deepening of the mixed layer leads to significant changes in the heat budget where the heat entrainment becomes the dominant heat sink, compensating and even exceeding the additional heat gain due to increased solar radiation. These different contributions interact together in a rather complex way at both local and basin scales, and despite weaker mean winds along the equator in the perturbed experiment, the deepening of the MLD is significant. In addition, the larger thermal inertia associated with the deeper mixed layer tends to reduce SST variations: SSTs higher than $29^{\circ} \mathrm{C}$ within the warm pool are thus no more maintained along the equator in the perturbed experiment. The cooler SSTs in turn affect the intensity of the atmospheric deep convection, therefore contributing to the changes in the mean atmospheric circulation. As mentioned in the previous section, such equilibrium is reached after a short period (less than 2 years) with no more drift noticed during the following 

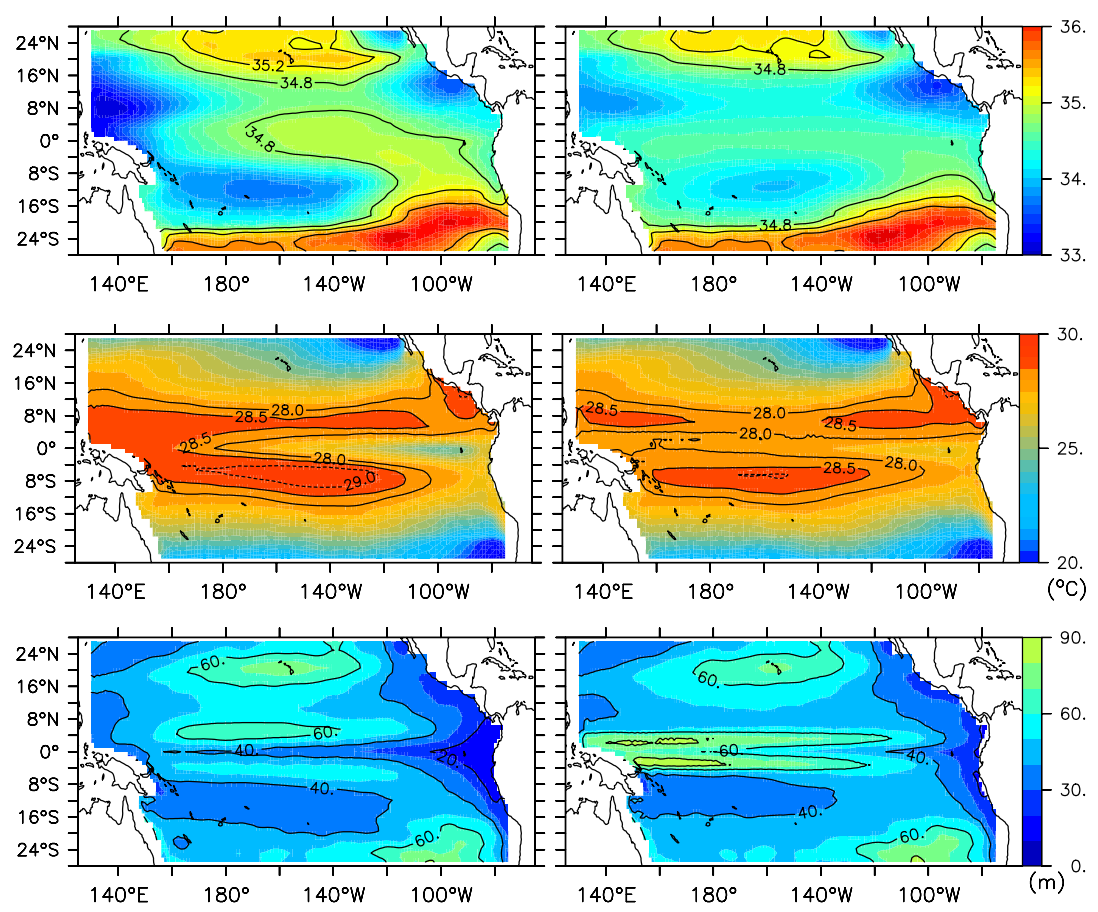

Fig. 2. Annual mean fields of SSS (a-top), SST (b-middle), and MLD (c-bottom) for the control (left) and perturbed (right) experiments. Contour interval is set to $0.4,0.5^{\circ} \mathrm{C}$, and $20 \mathrm{~m}$, respectively.

decades of the integration in the perturbed experiment.

Not surprisingly, changes in the time mean state of the oceanatmosphere coupled system also modify both the seasonal cycle and low frequency variability in the perturbed experiment. The seasonal cycle of the equatorial SSS is completely removed, with a zonal uniform value around 34.4 across the whole basin. Contrasting results are obtained for the SST field, that exhibits a weak seasonal cycle with peak-to-peak amplitude of $1.5^{\circ} \mathrm{C}$ in the eastern Pacific between the cold and warm seasons, with the phase being relatively unchanged when compared to the control experiment. As noted previously, there are also no more warm SSTs in the western Pacific in the perturbed experiment, especially during the boreal winter. This fact explains that neither weak westerly wind regime nor westerly wind bursts are simulated in the perturbed experiment, in contrast to the control experiment. All these modifications also alter the interannual variability of the coupled system. Spectral analysis of the SST anomalies relative to the seasonal cycle in the Nino3.4 region $\left(5^{\circ} \mathrm{N}-5^{\circ} \mathrm{S}, 120^{\circ} \mathrm{W}-\right.$ $170^{\circ} \mathrm{W}$ ) indicates that the amplitude in the spectral density major peak is significantly reduced between the experiments $(12 \%$ of the interannual variability near the 4 -yr period as compared to $24 \%$ near 3.6-yr period for the control experiment). Different statistical tests, considering either the global variance or the individual (per frequency band) variance, demonstrate that the differences between these spectra are significant at the 95\% confidence level. Figure 4 displays the temporal evolution of the SST anomalies along the equator for the first 15 years of both the control and perturbed experiments. One may notice that the low-frequency variability does not completely disappear in the perturbed experiment (rms of the Nino3.4 index decreases to $0.20^{\circ} \mathrm{C}$ as compared to $0.48^{\circ} \mathrm{C}$ for the control), as warm and cold anomalies with an amplitude of about $1{ }^{\circ} \mathrm{C}$ and lasting 6 to 8 months occur in the far eastern Pacific. Nevertheless, the amplitude of the anomalies, their duration in time and their zonal extension along the equator are clearly more developed in the control experiment, in much better agreement with the present observed features of ENSO.

\section{Conclusion and perspectives}

In the tropical oceans, the parameter that affects directly the

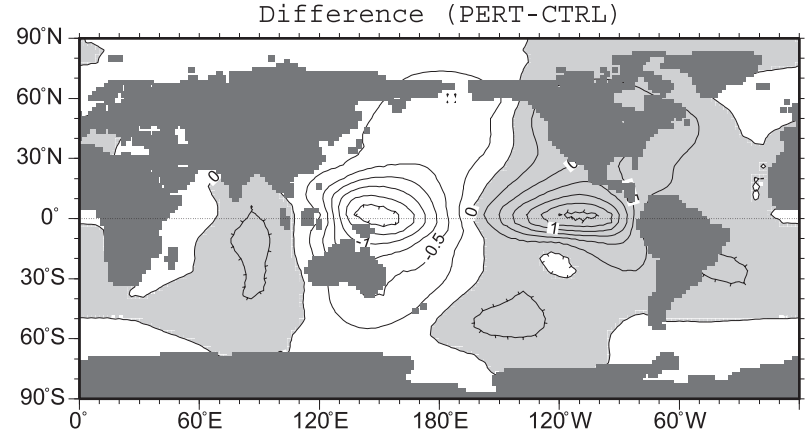

Fig. 3. Difference for the annual mean $1000 \mathrm{hPa}$ potential velocity of the atmosphere (in $10^{6} \mathrm{~m}^{2} \mathrm{~s}^{-1}$ ) between the perturbed and the control experiments. Positive values are shades.

atmospheric circulation is the SST. The processes responsible for the variability of this field are however various, and they involve not only the exchanges with the atmosphere, but also the dynamics and thermodynamics of the oceanic upper layers. Indeed, observations reported by Lukas and Lindstrom (1991) reveal that the situation is more complex in the equatorial western Pacific Ocean where the bottom of the ocean mixed layer depends on salinity stratification. This stratification is responsible for the cutoff of the cooling by the heat entrainment at the base of the mixed layer. To establish the role of the barrier layer in the feedbacks between the ocean and the atmosphere, it must be shown that its formation and presence both affect the SST. The present experiments complete previous studies reported by Maes et al. $(2002,2005)$, as they address these possibilities using a coupled GCM that was shown to do a reasonably good job in simulating the variability of the barrier layer and ENSO. Note that it would be worthwhile to conduct similar experiments with other coupled models because CGCMs are likely to be affected by different types of bias, and because of nonlinearities in the ocean-atmosphere coupled system. In that sense, it is very encouraging to see that Yim et al. (2008) described results that were similar to and coherent with ours, but using a different CGCM. 

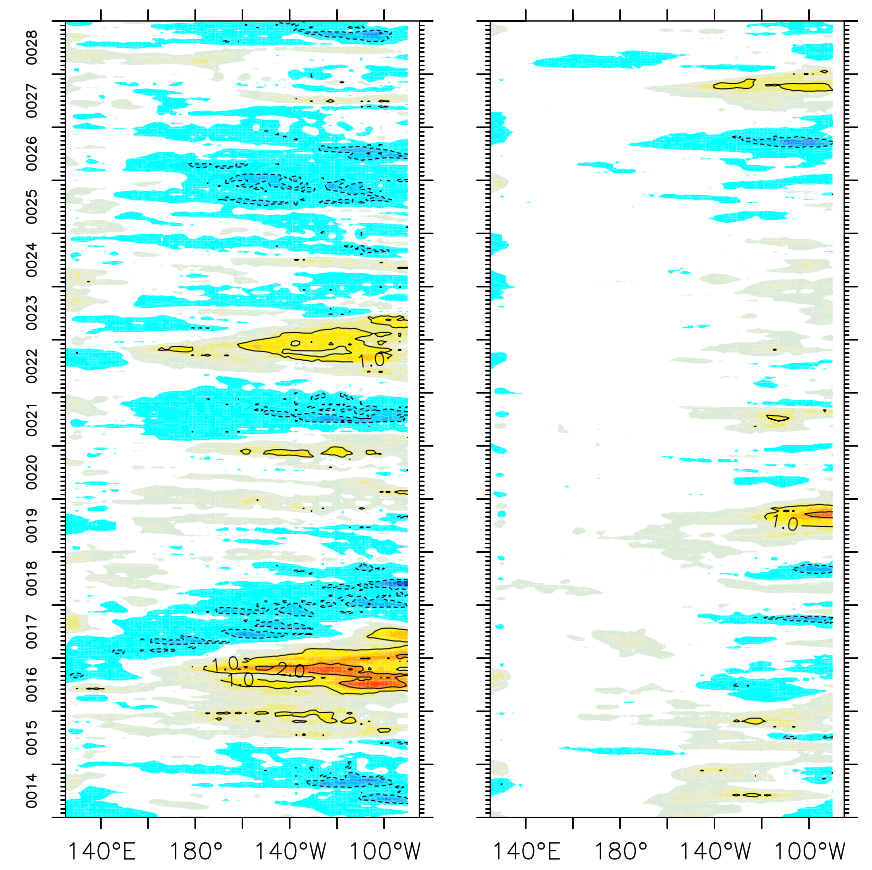

Fig. 4. Time-longitude diagram of SST anomalies along the equator for the control (left) and perturbed (right) experiments. The time axis covers a 15 -year long period. Contour interval is $1{ }^{\circ} \mathrm{C}$ and dashed lines correspond to negative values.

The western equatorial Pacific Ocean is of primary importance in the climate system through its role in the deep organized atmospheric convection, in the circulation and stratification of the ocean upper layers and, ultimately, in ENSO variability. Among the numerous atmospheric or oceanic processes that directly affect the SST variability, barrier layer occurrence is clearly of great importance. The main impact of the $\mathrm{BL}$ is to maintain a shallow mixed layer that may enhance the coupled response of the ocean-atmosphere system (typically resulting in a larger fetch for westerly wind bursts). Among all the diagnostics performed in the different sensitivity experiments operated with the present coupled model, this result is the most robust. Our previous results (Maes et al. 2002, 2005) showed that the BLT variability was able to modify the onset and/or the heat buildup prior to ENSO events. Beyond, the present study illustrates how BL formation is a key mechanism in establishing the time mean state of the Pacific Ocean, and further in setting background conditions that impact the low frequency variability of the coupled system.

It is important for both models and data assimilation methods that are used for seasonal-to-interannual forecasting issues to be able to reproduce the barrier layer variability realistically. Coupled ocean-atmosphere models must be able to produce westerly wind bursts as well as to generate the potentially resulting ocean barrier layer. They also have to reproduce the feedback between the fetch of such wind conditions along the equator and the characteristics of the SST field (presence and displacement of the warmest SSTs) within the warm pool. We do not believe that the aforementioned processes represent numerical artefacts because they share some common features with observations. The eastern edge of the warm pool whose zonal displacements may be associated with the formation of the barrier layer and with ENSO (Maes et al. 2004; Ando and Hasegawa 2009) represents the key region for both physical oceanography and biogeochemistry issues. Oceanographic cruises, dedicated specifically to this particular item, indeed reveal that salinity stratification is not only strongly related to the eastern edge of the warm pool (Maes 2008) but also affects biogeochemical variables (Matsumoto and Ando 2009; Maes et al. 2010). It is thus expected that we will be provided in the next few years with sufficient ocean in situ data to complete our knowledge of both the barrier layer and its relationship with ENSO, in order to be able to take into account properly such processes in earth's climate forecast systems.

\section{Acknowledgements}

The authors would like to thank the French community in charge of the atmospheric (ARPEGE model from Météo-France), oceanic (NEMO model from LOCEAN), and coupling (OASIS from CERFACS) components of the coupled model used in the present study. Computer facilities were provided by Météo-France and by the Centre National d'Etudes Spatiales. The authors gratefully acknowledge Joël Picaut, Jay McCreary and Dave Behringer for their comment and support. This work was supported by the Programme National d'Etudes de la Dynamique du Climat and by the Institut de Recherche pour le Développement.

\section{References}

Ando, K., and M. J. McPhaden, 1997: Variability of surface layer hydrography in the tropical Pacific Ocean. J. Geophys. Res., 102, 2306323078 .

Ando, K., and T. Hasegawa, 2009: Annual zonal displacement of Pacific warm pool in association with El Niño onset. SOLA, 5, 149-152, doi:10.2151/sola.2009-038.

Belamari, S., J.-L. Redelsperger, and M. Pontaud, 2003: Dynamic role of a westerly wind burst in triggering an equatorial Pacific warm event. $J$. Climate, 16, 1869-1890.

Bosc, C., T. Delcroix, and C. Maes, 2009: Barrier layer variability in the western Pacific warm pool from 2000 to 2007. J. Geophys. Res., 114, C06023, doi:10.1029/2008JC005187.

Collins, M., S.-I. An, W. Cai, A. Ganachaud, E. Guilyardi, F.-F. Jin, M. Jochum, M. Lengaigne, S. Power, A. Timmermann, G. Vecchi, and A. Wittenberg, 2010: The impact of global warming on the tropical Pacific Ocean and El Niño. Nature Geoscience, 3, 391-397, doi: $10.1038 /$ ngeo868.

Cronin, M. F., and M. J. McPhaden, 2002: Barrier layer formation during westerly wind bursts. J. Geophys. Res., 107(C12), 8020, doi:10.1029/ 2001JC001171.

Delecluse, P., M. Davey, Y. Kitamura, S. Philander, M. Suarez, and L. Bengtsson, 1998: Coupled general circulation modelling of the tropical Pacific. J. Geophys. Res., 103(C7), 14357-14373.

Lukas, R., and E. Lindstrom, 1991: The mixed layer of the western equatorial Pacific Ocean. J. Geophys. Res., 96, 3343-3357.

Maes, C., 2008: On the ocean salinity stratification observed at the eastern edge of the equatorial Pacific warm pool. J. Geophys. Res., 113, C03027, doi:10.1029/2007JC004297.

Maes, C., J. Picaut, and S. Belamari, 2002: Salinity barrier layer and onset of El Niño in a Pacific coupled model. Geophys. Res. Lett., 29(24), 2206, doi:10.1029/2002GL016029.

Maes, C., J. Picaut, Y. Kuroda, and K. Ando, 2004: Characteristics of the convergence zone at the eastern edge of the Pacific warm pool. Geophys. Res. Lett., 31, L11304, doi:10.1029/2004GL019867.

Maes, C., J. Picaut, and S. Belamari, 2005: Importance of salinity barrier layer for the buildup of El Niño. J. Climate, 18, 104-118.

Maes, C., J. Sudre, and V. Garçon, 2010: Detection of the eastern edge of the equatorial Pacific warm pool using satellite-based ocean color observations. SOLA, 6, 129-132, doi:10.2151/sola.2010-033.

Matsumoto, K., and K. Ando, 2009: Use of cyanobacterial pigments to characterize the ocean surface mixed layer in the western Pacific warm pool. J. Mar. Syst., 75, 245-252.

Picaut, J., M. Ioualalen, C. Menkes, T. Delcroix, and M. J. McPhaden, 1996: Mechanism of the zonal displacements of the Pacific warm pool: implications for ENSO. Science, 274, 1486-1489.

Roemmich, D., M. Morris, W. R. Young, and J.-R. Donguy, 1994: Fresh equatorial jets. J. Phys. Oceanogr., 24, 540-558.

Yim, Y., S.-W. Yeh, Y. Noh, B.-K. Moon, and Y.-G. Park, 2008: Sea surface salinity variability and its relation to El Niño in a CGCM. AsiaPacific J. Atm. Sci., 44, 173-189.

Manuscript received 18 April 2011, accepted 14 June 2011

SOLA: http://www.jstage.jst.go.jp/browse/sola 